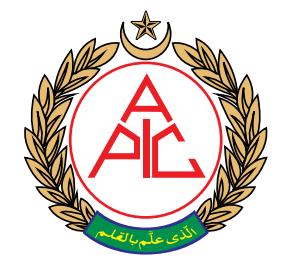

Department of Anesthesiology \& Intensive Care, Universiti Kebangsaan Malaysia Medical Center. Jalan Yaakob Latif, 56000 Kuala Lumpur, Malaysia

Correspondence:

Dr Syarifah Noor Nazihah

Sayed Masri, Dr Anaes \& Crit

Care (UKM, Malaysia)

Department of Anesthesiology

\& Intensive Care,

Pusat Perubatan Universiti

Kebangsaan Malaysia,

Jalan Yaakob Latif, 56000

Kuala Lumpur, Malaysia.

Phone: +60 133452456

E-mail:

syarifahnazihah@yahoo.com. my

Received - 6 August 2019,

Reviewed -21 March 2020 ,

Revised - 9 March 2020,

Accepted - 22 March 2019

\section{Accuracy of digital palpation and laryngeal handshake in identification of cricothyroid membrane: An observational study}

Khor Whuan Wyeen, Syarifah Noor Nazihah Sayed Masri, Aliza Mohammad Yusof, Azarinah Izaham, Siti Nidzwani Mohammad Mahdi, Nadia Md Nor

\section{ABSTRACT}

Background: Evaluation of the anterior neck anatomy is essential to identify the cricothyroid membrane (CTM) before invasive surgical airway. This study aimed to compare the accuracy of cricothyroid membrane identification done by digital palpation (DP) method and laryngeal handshake palpation (LHP).

Methodology: A total of 52 participants were recruited to perform DP and LHP procedures on study patients. Using fluorescent "invisible" ink, the participants were asked to mark the cricothyroid membrane. The actual level of CTM was identified using ultrasound by the investigator prior to the land marking and the distance between the actual and estimated margin of the CTM was measured. Time taken to identify CTM and the ease of palpation was measured using a 3-point Likert scale.

Results: There were 52 patients in total, with 26 obese and equal number of nonobese patients. CTM was correctly identified in $78.8 \%$ of cases using both methods. There was no statistically significant difference between DP and LHP in terms of absolute distance to the midline of CTM $(p=0.361)$, time to CTM identification $(p=0.101)$ and ease of palpation $(p=0.059)$. The time required to identify the CTM in male patients were shorter when using the laryngeal handshake technique.

Conclusion: Both digital palpation and laryngeal handshake palpation techniques are comparable in localizing cricothyroid membrane in obese and non-obese patients.

Key words: Cricothyroidotomy; Cricothyroid membrane; CTM landmarking; Digital palpation; Laryngeal handshake palpation

Citation: Wyeen KW, Masri SNNS, Yusof AM, Izaham A, Mahdi SNMM, Nor NM. Accuracy of digital palpation and laryngeal handshake in identification of cricothyroid membrane: An observational study. Anaesth. pain intensive care 2020;24(1):168-174 DOI: https://doi.org/10.35975/apic.v24i2.1253

\section{INTRODUCTION}

Emergency cricothyroidotomy is a life-saving maneuver that may prevent death or permanent neurological injury in the event of difficult or failed airway management. A 'can't intubate, can't oxygenate' (CICO) situation is declared when attempts to manage airway within stipulated conditions via face mask, supraglottic device or tracheal intubation have failed and cricothyroidotomy is preformed immediately as a life-saving procedure. ${ }^{1}$ This scenario may be unexpected and accounts for $25 \%$ of anesthesia related deaths. ${ }^{1}$ A successful cricothyroidotomy depends upon the correct identification of the cricothyroid membrane (CTM), as a misplaced 
incision in the membrane risks damaging surrounding vascular structures and delays the safe completion of procedure. ${ }^{2}$ However, cricothyroidotomy is an infrequently performed procedure with complication rates ranging from $9 \%$ to $40 \%{ }^{3}$

Identification of CTM performed using digital palpation (DP) technique has an accuracy of up to $70 \%{ }^{2}$ The laryngeal handshake palpation (LHP) as described by Levitan, was recommended by the Difficult Airway Society (DAS) in 2015 as the first step in locating CTM. ${ }^{3,4}$ The laryngeal handshake promotes confidence by giving a better appreciation of the three dimension laryngeal structures and stabilization of the larynx, which also makes an easier identification of CTM. Identification of CTM had accuracy of $63.4 \%$ for DP and $70.7 \%$ for LHP in a study by Oh et al. ${ }^{3,5}$ Another study by T. Drew et al. showed accuracy of 33\% for DP and $62 \%$ for LHP. ${ }^{6}$

We conducted this study to compare the accuracy and speed of DP and LHP in identifying the CTM amongst anesthesia practitioners of our institution.

\section{METHODOLOGY}

This observational study was conducted after obtaining approval from the Research Committee of Department of Anesthesiology \& Intensive Care, Faculty of Medicine, Hospital Canselor Tuanku Mukhriz (HCTM), Universiti Kebangsaan Malaysia Medical Centre (UKMMC) UKM vie their letter No. PPI/111/8/JEP-2017-475. Further approval was also obtained from the Medical Research \& Ethics Committee UKMMC and the Medical Research \& Ethics Committee (MREC) under National Medical Research Register (NMRR16-2751-33736), Ministry of Health Malaysia.

This study was conducted in Hospital Kuala Lumpur from January 2018 to November 2018. A written informed consent obtained from the patients and participants who were involved in this study by a single investigator. The patients were recruited during pre-anesthetic clinic or premedication visit and depending on the place of the recruitment, the identification of CTM was performed either in clinic or operating room prior to induction of anesthesia. Less than $18 \mathrm{y}$ old individuals, pregnant ladies, those with a known neck deformity, neck swelling or cervical spine problem, previous neck surgery or irradiation were excluded from the study. The patient's demographic data, including age, gender, height, weight and body mass index (BMI), was documented. Patients were further sub-divided into two equal groups according to their BMI; < $30 \mathrm{~kg} / \mathrm{m}^{2}$ (non-obese) and $\geq 30$ $\mathrm{kg} / \mathrm{m}^{2}$ (obese). The airway parameters (neck circumference, thyro-mental and sterno-mental distance) were measured with the ribbon measuring tape and recorded.

Each patient was positioned supine and neck was positioned in extension by placing a pillow underneath the shoulders. The sole investigator, who had undergone training in neck ultrasonography as described by Kristensen ${ }^{7}$ (Figure 1), scanned for CTM using ultrasound machine (SonoSite Inc., Boothell, WA, USA). The landmark marking technique was based upon description provided by You-Ten et al. with a slight variation. ${ }^{8}$ The linear high frequency ultrasound transducer was placed transversely just above the suprasternal notch to demonstrate the trachea. Afterwards, the right end of transducer was moved medially to locate the midline of trachea. Once the right end of transducer was in the midline, the left end of the probe was rotated into the sagittal plane to produce longitudinal scan of the midline of trachea. The transducer was then moved cranially in the midline to see the cricoid cartilage, CTM and thyroid cartilage. A 2-cm vertical line was drawn using the invisible-ink pen (Security Magic Invisible Ink Marker UV Spy Pen, China) of which the markings could be seen with ultraviolet light exposure. The horizontal midline, superior and inferior borders of CTM were determined by sliding the $27 \mathrm{G}$ intravenous catheter under and perpendicular to the transducer to create the drop-out shadow effect on the relevant CTM borders. These landmarks were subsequently marked with the invisible-ink pen. The point where the vertical line intersected with the horizontal midline was 

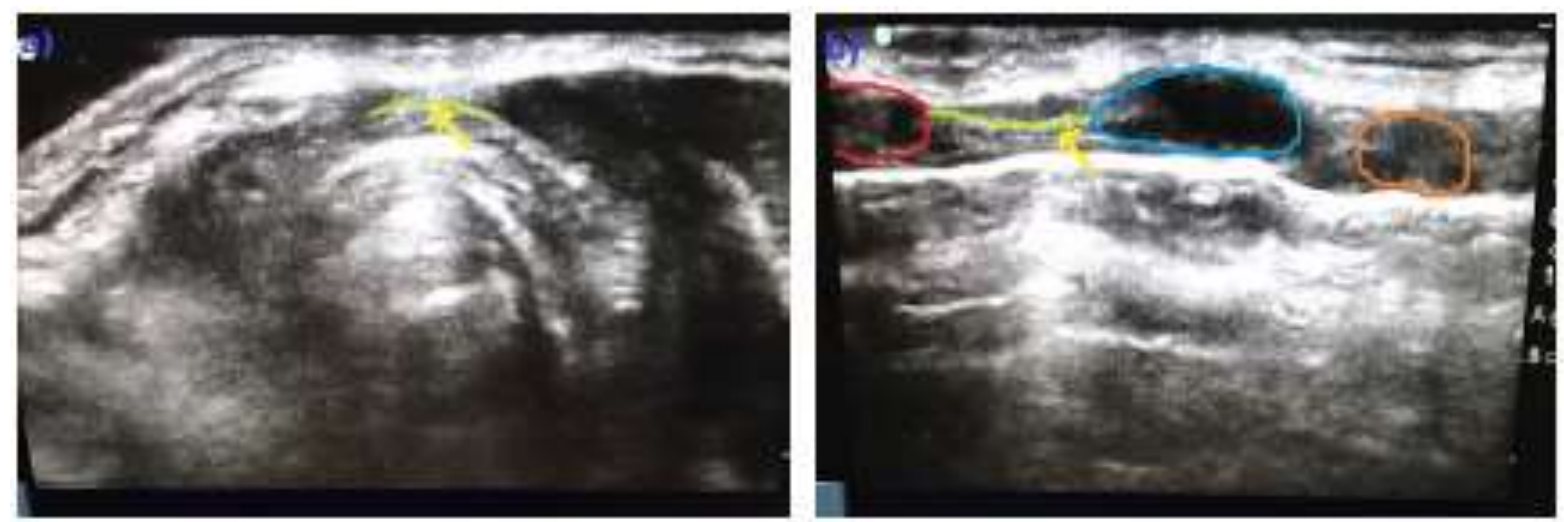

Figure 1(a): Transverse view of ultrasound showing the arch-like appearance of CTM (green line with arrow), (b) The sagittal view showing the thyroid cartilage (red), cricoid cartilage (blue), tracheal ring (orange) and CTM (green line with arrow).

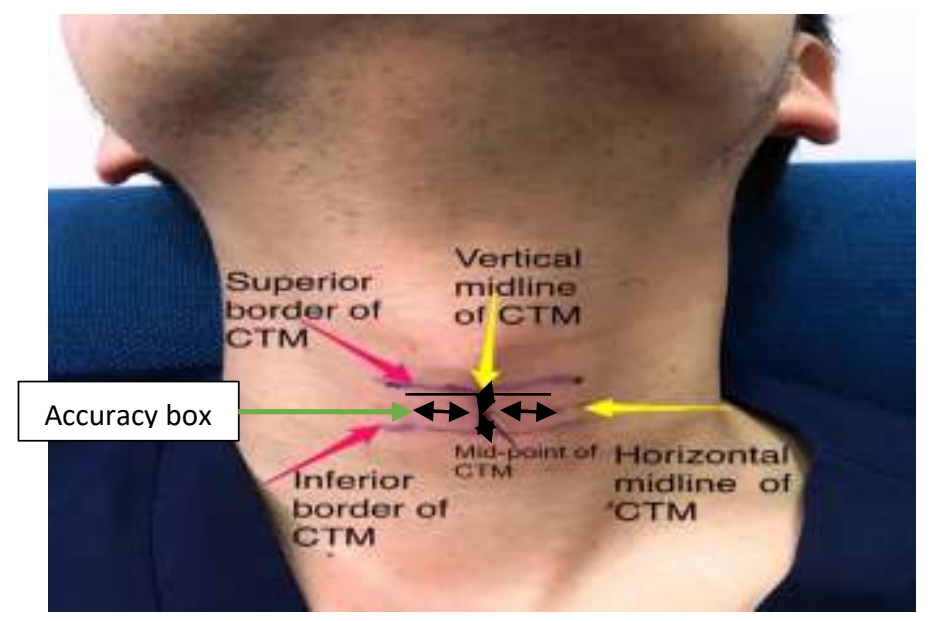

Figure 2: Picture showing the landmarks of CTM guided by ultrasound scan
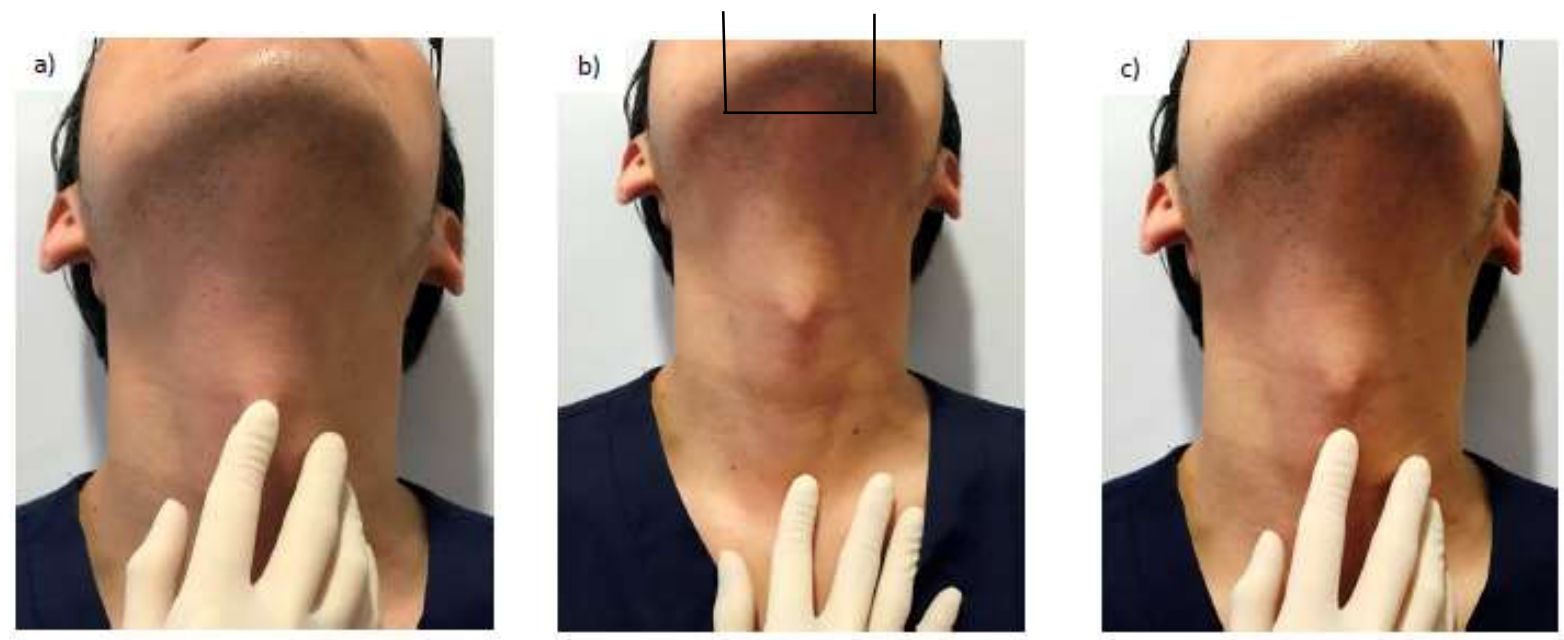

Figure 3: Digital palpation technique. Palpation either starting from (a) Thyroid notch, or (b) Sternal notch, to the (c) Cricothyroid membrane 

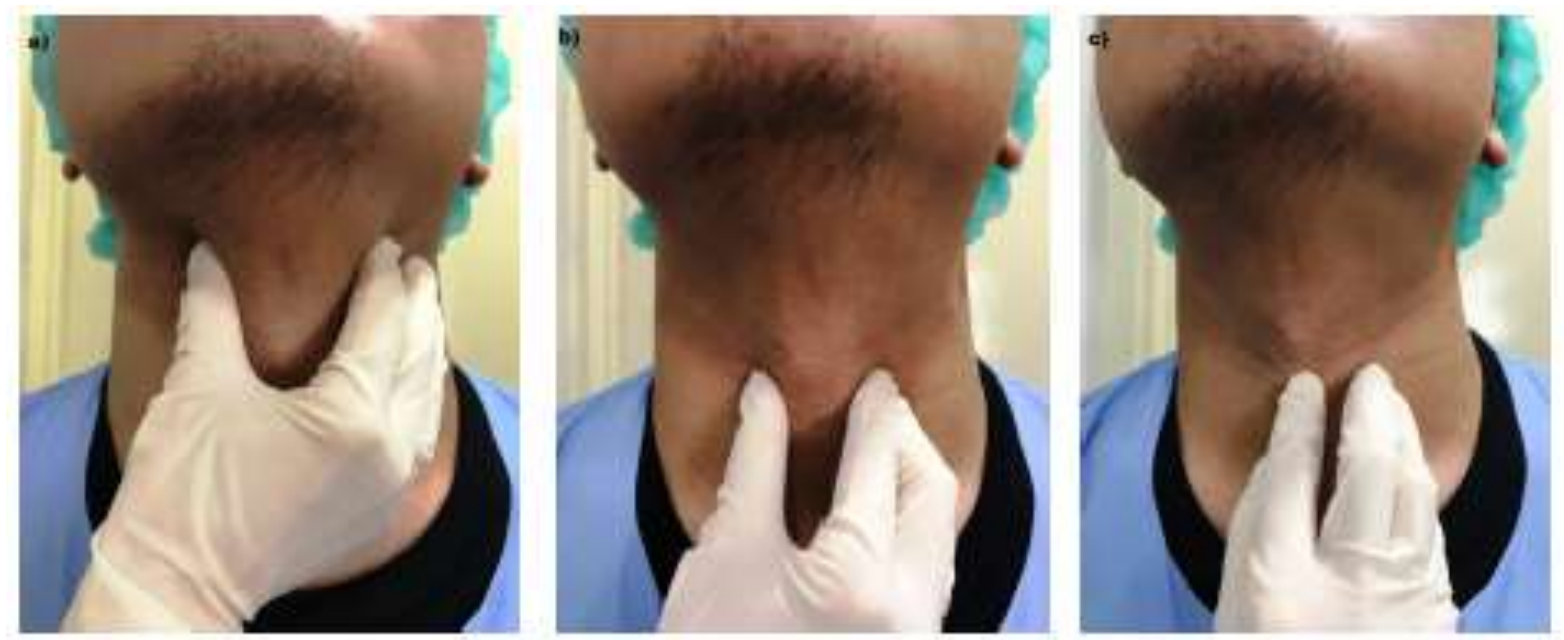

Figure 4: The laryngeal handshake. (a) The thumb and index finger grasp the greater cornu of the hyoid bone (top of the larynx) and roll it from side to side. (b) The fingers and thumb slide down over thyroid laminae. (c) Middle finger and thumb rest on the cricoid cartilage with the index finger palpating for cricothyroid membrane

taken as the mid-point of CTM. An accuracy box was then defined as $0.5 \mathrm{~cm}$ from mid-point, vertically and horizontally (Figure 2).

Participants consisted of anesthesia service medical officers, postgraduate anesthesia trainees and specialists. Anesthesiology experience in years for each participant was documented. Each participant performed both techniques (DP and LHP) on one patient. Each patient was palpated once by a participant. Before performing the technique, a briefing on both techniques was given to all the participants (Figure 3 and 4). Marking was done using 'dot' and 'cross' shapes for DP and LHP techniques respectively.

The absolute distance was defined as the distance from the mark to the mid-point of CTM created by ultrasound. It was measured using ribbon measuring tape and recorded. The accuracy of palpation was defined as marking within the accuracy box. If the marking was accurate, the location of the mark, whether placed above, below or at the horizontal midline of CTM was documented. The palpation time, time taken from the time of palpation to the completion of marking on the neck, was measured using a stopwatch (Casio HS-3V-1,
Casio Computer Co., Japan) and recorded. Lastly, the participants used the 3-point Likert scale to rate the ease of palpation. Options given to them were easy (clear visible landmarks), moderate (light palpation) or difficult (deep palpation).

\section{Statistical analysis:}

The study was designed with type I error of alpha 0.05 , type II error of beta 0.2 and power of $80 \%$. Based on study by Aslani et al. ${ }^{9}$ that showed $24 \%$ accuracy in identifying the cricothyroid membrane and the assumption that a $70 \%$ accuracy would be clinically meaningful when the laryngeal handshake method is used, ${ }^{5}$ the calculated sample size was 52 , with $20 \%$ dropout rate.

Data were analyzed using IBM SPSS statistics, version 23 (IBM ${ }^{\mathrm{TM}}$ Corp. Armonk, USA). We analyzed the categorical values using numbers or percentage and the continuous values using mean with standard deviation or median with range. For intragroup (DP and LHP) analysis, Wilcoxon signed rank test were used to compare the absolute distance, palpation time and ease of palpation. McNemar test was employed to compare the accuracy of palpation. A value of $p$ $<0.05$ was considered statistically significant. 


\section{RESULTS}

A total of 52 patients and 52 participants were enrolled in the study. Table 1 presents the characteristics of patients and participants. Table 2 and 3 tabulates the CTM profiles between DP and LHP methods. Both methods had same success rate where $78.8 \%$ of cases were correctly identified.

There was no statistically significant difference between DP and LHP in term of accurate identification of the midline $(p=0.999)$, time to CTM identification $(p=0.101)$ and ease of palpation $(p=0.059)$. In male patients, significantly shorter time was taken to identify CTM using LHP as compared to DP method ( $p$ $=0.024)$. Participants with more than 5 years of working experience found it easier to identify CTM using DP method.

In terms of accurate identification, this study observed an almost perfect agreement between DP and LHP method in all subjects (Kappa = $0.89, p<0.001)$. Subgroup analysis also showed good agreement between methods among obese and non-obese subjects (Kappa $=0.91, p<$ 0.001 ; Kappa $0.84, p<0.001$, respectively) (Table 4).

\section{DISCUSSION}

The results from our study showed similar accuracy of $78 \%$ in successful identification of CTM between digital palpation technique and laryngeal handshake palpation. In a recently published study, Oh et al..$^{5}$ reported the success rate of identifying the cricothyroid membrane was similar among the anesthesiologists who performed the laryngeal handshake method and among otorhinolaryngologists who used simple palpation (LHP 70.7\%, DP 63.4\%). In contrast, T. Drew et $a l .{ }^{6}$ who included only female subjects, showed that laryngeal handshake palpation was more accurate but took longer than conventional palpation technique in locating the CTM and the midline (LHP 62\%, DP $33 \%$ ).
Obesity and gender are the two commonly studied factors that may influence the identification of the cricothyroid membrane. Our study found that the accuracy of CTM identification were similar between obese and non-obese subjects. This differs with findings from previous studies where increased neck circumference in obese patients was associated with inaccuracy in locating the CTM. ${ }^{5,9}$ In a previous study on the influence of obesity on successful identification of the CTM, a success rate of $3.3 \%$ in obese females compared to $26.8 \%$ in non-obese females was found. ${ }^{9}$

It is well established that palpation of CTM is more accurate in male compared to female gender. Campbell et al. demonstrated that success rate of identification of CTM was $69.4 \%$ in male compared to $19.4 \%$ in female. ${ }^{10}$ The discrepancy may be due to inclusion of males who have more prominent anatomical landmarks. This was further supported by findings from a large cadaveric study showing that the mean vertical size of the cricothyroid ligament was shorter in females $(6.4 \mathrm{~mm})$ compared to male specimens $(7.6 \mathrm{~mm}){ }^{11}$ Thus, the likely mechanism for this inter-gender difference is the shape and size of the adjacent laryngeal cartilage. ${ }^{11,12,13,14}$ Both the vertical height and width of the CTM itself are greater in males which provide the clinician a larger target to locate. ${ }^{15}$

There have been a number of alternative techniques proposed for CTM identification at the bedside, none of which are sufficiently accurate to recommend them for clinical use. ${ }^{16}$ These include an approximation based on four finger widths and a method based on skin creases present on the anterior neck. They has an accuracy of $46 \%$ and $50 \%$ respectively, when performed by emergency physicians with formal training in airway techniques. ${ }^{2}$ The combined results of the mentioned observational studies and the poor performance of other bedside methods are all strong arguments for the development of alternative strategies. 
We found that the highest proportion of misidentified sites were marked below the CTM. This is comparable with results from $\mathrm{T}$ Drew et al. ${ }^{6}$ Theoretically, this would have resulted in tracheal access but at the potential cost of posterior tracheal wall and esophageal injury, which has been associated with both scalpel and trocar techniques. ${ }^{3,16}$ Airway access attempts above the CTM are unlikely to achieve airway patency. Devices inserted lateral to the midline are more likely to cause major vascular injuries because of the proximity of the carotid artery and jugular venous systems.

\section{LIMITATIONS}

There are a few limitations in this study. This study was performed under optimal circumstances in a relaxed, non-emergent setting. In reality, cricothyroidotomy for failed airway management is an emergency procedure and is potentially fraught with anxiety and error. The CTM identification attempts were done on conscious patients. This is unlike the studies conducted by $\mathrm{Oh}$ et al..$^{5}$ and T. Drew et al. ${ }^{6}$ where patients were under general anesthesia, as in a real life CICO scenario. The laryngeal handshake requires a grip and roll of the cartilaginous airway structures which can be uncomfortable and participants may be reluctant to perform this to full effect on conscious patients

\section{CONCLUSIONS}

Both DP and LHP techniques are comparable for identifying CTM in obese and non-obese patients. However, the time required to identify the CTM in male patients was shorter using the laryngeal handshake method. Whereas, participants with more than 5 years of working experience perceived DP to be easier for identifying CTM. We believe refresher courses should be reinforced by regular practice, because such skills are likely to deteriorate when not preformed frequently. Additionally, marking of the CTM by ultrasound before induction of anesthesia may also be of value in patients on high risk for failed intubation.

\section{Conflict of interest:}

None declared by the authors

\section{Authors' contribution:}

KWW: Conduction of the study work, data analysis, manuscript writing

SNNSM: Data analysis, manuscript editing

AMY: Concept

SNMM, NMN: Manuscript editing

Al: Supervision, manuscript editing

\section{REFERENCES}

1. Ferk $C$, Mitchell VS, McNarry AF, Mendonca $C$, Bhagrath $R$, Patel A, et al. Difficult airway society 2015 guidelines for management of unanticipated difficult intubation in adults. $\mathrm{Br} \mathrm{J}$ Anaesth. 2015;115(6):827-48. [PubMed] DOI: 10.1093/bja/aev371

2. Bair $A E$, Chima R. The inaccuracy of using landmark techniques for crictothyroid membrane identification: a comparison of three techniques. Acad Emerg Med. 2015;22:908-14. [PubMed] DOI: 10.1111/acem.12732

3. Siddiqui N, Arzola C, Friedman Z, Guerina L, You-ten KE. Ultrasound improves cricothyrotomy success in cadavers with poorly defined neck anatomy: a randomized control trial.
Anesthesiology. 2015;123:1033-41. [PubMed] DOI: 10.1097/ALN.0000000000000848

4. Yen C. Levitan. The "Laryngeal Handshake" and the "Cartilaginous Cage". Available from: http://prehospitalmed.com/2013/11/ 17/levitan-the-laryngeal-handshakeand-the-cartilaginous-cage [Accessed 17 November 2013]

5. Oh H, Yoon S, Seo M, Oh E, Yoon $H$, Lee $H$, et al. Utility of the laryngeal hand shake method for identifying the cricothyroid membrane. Acta Anaesthesiol Scand. 2018;62(9):1223-8. [PubMed] DOI: 10.1111/aas.13169

6. Drew T, McCaul CL. Laryngeal handshake technique in locating the cricothyroid membrane: a non randomised comparative study. $\mathrm{Br} \mathrm{J}$ Anaesth. 2018 Nov;121(5):1173-8. PubMed] DOI: 10.1016/j.bja.2018.07.034

7. Kristensen MS. Ultrasonography in the management of the airway. Acta Anaesthesiol Scand. 2011 Nov;55(10):1155-73. [PubMed] DOI: 10.1111/j.1399-6576.2011.02518.x

8. You-Ten KE, Desai D, Postonogava $T$, Siddiqui N. Accuracy of conventional digital palpation and ultrasound of the cricothyroid membrane in obese women in labour. Anaesthesia. 2015 Nov;70(11):1230-4. [PubMed] DOI: 10.1111/anae.13167

9. Aslani $\mathrm{A}, \mathrm{Ng} \mathrm{SC}$, Hurley $\mathrm{M}$, McCarthy KF, McNicholas M, 
McCaul CL.. Accurarcy of identification of the cricothyroid membrane in female subjects using palpation: An observational study. Anesth Analg. 2012 May;114(5):987-92. [PubMed] DOI: 10.1213/ANE.0b013e31824970ba

10. Campbell M, Shanahan H, Ash S, Royds J, Husarova V, McCaul C. The accuracy of locating the cricothyroid membrane by palpation-an intergender study. BMC Anesthesiol. 2014;14:108. [PubMed] DOI: 10.1186/1471-225314-108

11. Randestad A, Lindholm CE, Fabian $P$. Dimensions of the cricoid cartilage and the

trachea.

Laryngoscope.

2000;110:1957-61.

[PubMed] DOI: 10.1097/00005537200011000-00036

12. Jain $\mathrm{M}$, Dhall U. Morphometry of the thyroid and cricoid cartilages in adults. J Anat Soc India. 2008;57(2):119-23.

13. Ajmani ML. A metrical study of laryngeal skeleton in adult Nigerian. J Anat. 1990;171(1):187-91. [PubMed]

14. Jotz GP, Lealo HZQ, da Costa Filho $\mathrm{OP}$, Fisch $\mathrm{P}$, Magalhales $\mathrm{RC}$ Cervantes $\mathrm{O}$. The asymmetry index of the cricoid cartilage and the external angle of the thyroid cartilage. A sex-related study. Eur J Anat. 2007;11(1):1-7. [Free Full Text]

15. Long N, Ng S, Donnelly G, Owens $\mathrm{M}$, McNicholas M, McCarthy KF, et al. Anatomical characterization of the cricothyroid membrane in females of child bearing age using computer tomography. Int J Obstet Anesth. 2014;23(1):29-34.

[PubMed] $\mathrm{DOI}$ 10.1016/i.joa.2013.07.007

16. Salah N, El Saigh I, Hayes N, McCaul CL. Airway injury during emergency transcutaneous airway access: a comparison at cricothyroid and tracheal sites. Anesth Analg. 2009;109:1901-7. [PubMed] DOI: 10.1213/ANE.0b013e3181bdd1ba

17. Ferk C, Mitchell VS, McNarry AF, Mendonca C, Bhagrath R, Patel A, et al. Difficult airway society 2015 guidelines for management of unanticipated difficult intubation in adults. $\mathrm{Br} \mathrm{J}$ Anaesth. 2015;115(6):827-48. [PubMed] DOI: 10.1093/bja/aev371

18. Bair $A E$, Chima $R$. The inaccuracy of using landmark techniques for crictothyroid membrane identification: a comparison of three techniques. Acad Emerg Med. 2015;22:908-14. [PubMed] DOI: 10.1111/acem. 12732

19. Siddiqui N, Arzola C, Friedman Z, Guerina L, You-ten KE. Ultrasound improves cricothyrotomy success in cadavers with poorly defined neck anatomy: a randomized control trial. Anesthesiology. 2015;123:1033-41. [PubMed] DOI: 10.1097/ALN.0000000000000848

20. Yen C. Levitan. The "Laryngeal Handshake" and the "Cartilaginous Cage". Available from: http://prehospitalmed.com/2013/11/ 17/levitan-the-laryngeal-handshakeand-the-cartilaginous-cage [Accessed 17 November 2013].

21. Oh H, Yoon S, Seo M, Oh E, Yoon $H$, Lee $H$, et al. Utility of the laryngeal hand shake method for identifying the cricothyroid membrane. Acta Anaesthesiol Scand. 2018;62(9):1223-8. [PubMed DOI: 10.1111/aas.13169

22. Drew T, McCaul CL. Laryngeal handshake technique in locating the cricothyroid membrane: a non randomised comparative study. $\mathrm{Br} \mathrm{J}$ Anaesth. 2018 Nov;121(5):1173-8. [PubMed]

DOI: 10.1016/i.bja.2018.07.034

23. Kristensen MS. Ultrasonography in the management of the airway. Acta Anaesthesiol Scand. 2011 Nov;55(10):1155-73. [PubMed] DOI: 10.1111/j.1399-6576.2011.02518.x

24. You-Ten KE, Desai D, Postonogava $T$, Siddiqui N. Accuracy of conventional digital palpation and ultrasound of the cricothyroid membrane in obese women in labour. Anaesthesia. 2015 Nov;70(11):1230-4. [PubMed] DOI: 10.1111/anae.13167

25. Aslani A, Ng SC, Hurley M, McCarthy KF, McNicholas M,
McCaul CL.. Accurarcy of identification of the cricothyroid membrane in female subjects using palpation: An observational study. Anesth Analg. 2012 May;114(5):987-92. [PubMed] DOI: 10.1213/ANE.0b013e31824970ba

26. Campbell M, Shanahan H, Ash S, Royds J, Husarova V, McCaul C. The accuracy of locating the cricothyroid membrane by palpation-an intergender study. BMC Anesthesiol. 2014;14:108. [PubMed] DOI: 10.1186/1471-225314-108

27. Randestad A, Lindholm CE, Fabian $P$. Dimensions of the cricoid cartilage and the trachea. Laryngoscope. 2000;110:1957-61. [PubMed] DOI: 10.1097/00005537200011000-00036

28. Jain M, Dhall U. Morphometry of the thyroid and cricoid cartilages in adults. J Anat Soc India. 2008;57(2):119-23.

29. Ajmani ML. A metrical study of laryngeal skeleton in adult Nigerian. J Anat. 1990;171(1):187-91. [PubMed]

30. Jotz GP, Lealo HZQ, da Costa Filho $\mathrm{OP}$, Fisch $\mathrm{P}$, Magalhales RC, Cervantes $\mathrm{O}$. The asymmetry index of the cricoid cartilage and the external angle of the thyroid cartilage. A sex-related study. Eur J Anat. 2007;11(1):1-7. [Free Full Text]

31. Long N, Ng S, Donnelly G, Owens $\mathrm{M}, \mathrm{McNicholas} \mathrm{M}, \mathrm{McC}$ arthy KF , et al. Anatomical characterization of the cricothyroid membrane in females of child bearing age using computer tomography. Int J Obstet Anesth. 2014;23(1):29-34. [PubMed] DOl: 10.1016/j.ijoa.2013.07.007

32. Salah N, El Saigh I, Hayes N, McCaul CL. Airway injury during emergency transcutaneous airway access: a comparison at cricothyroid and tracheal sites. Anesth Analg. 2009;109:1901-7. [PubMed] DOI: 10.1213/ANE.0b013e3181bdd1ba 